\title{
Object Individuation or Object Movement as Attractor? A Replication of the Wide-Screen/Narrow-Screen Study by Means of (a) Standard Looking Time Methodology and (b) Eye Tracking
}

\author{
Peter Krøjgaard, ${ }^{1}$ Osman S. Kingo, ${ }^{2}$ and Søren R. Staugaard ${ }^{1}$ \\ ${ }^{1}$ Center on Autobiographical Memory Research, Department of Psychology and Behavioural Sciences, Aarhus University, \\ Bartholins Allé 9, 8000 Aarhus C, Denmark \\ ${ }^{2}$ Center on Autobiographical Memory Research, Department of Psychology and Behavioural Sciences and MINDLab, \\ Aarhus University, Bartholins Allé 9, 8000 Aarhus C, Denmark
}

Correspondence should be addressed to Peter Krøjgaard; peter@psy.au.dk

Received 18 March 2013; Accepted 23 June 2013

Academic Editor: Andrew N. Meltzoff

Copyright (C) 2013 Peter Krøjgaard et al. This is an open access article distributed under the Creative Commons Attribution License, which permits unrestricted use, distribution, and reproduction in any medium, provided the original work is properly cited.

\begin{abstract}
We report a replication experiment of a mechanized version of the seminal wide-screen/narrow-screen design of Wilcox and Baillargeon (1998) with 9.5-month-old infants $(N=80)$. Two different methodologies were employed simultaneously: (a) the standard looking time paradigm and (b) eye tracking. Across conditions with three different screen sizes, the results from both methodologies revealed a clear and interesting pattern: the looking times increased as a significantly linear function of reduced screen sizes, that is, independently of the number of different objects involved. There was no indication in the data that the infants made use of the featural differences between the different-looking objects involved. The results suggest a simple, novel, and thoughtprovoking interpretation of the infants' looking behavior in the wide-screen/narrow-screen design: moving objects are attractors, and the more space left for visible object movement in the visual field, the longer are infants' looks. Consequently, no cognitive interpretation may be needed.
\end{abstract}

\section{Introduction}

Object individuation refers to the process of deciding the number of distinct objects present in a given scenario (for reviews, see $[1,2])$. In a now seminal paper, $\mathrm{Xu}$ and Carey [3] provided evidence that 10 -month-old infants could only successfully individuate objects if they were given unequivocal spatiotemporal information about the number of objects present, whereas featural information was insufficient. Shortly after, Wilcox and Baillargeon [4] argued that the results obtained by $\mathrm{Xu}$ and Carey [3] could have been a consequence of the cognitively demanding design employed, rather than a general inability to use featural information when attempting to individuate objects. In order to substantiate their claim, Wilcox and Baillargeon [4] devised a simpler version of the design originally used by $\mathrm{Xu}$ and Carey-the so-called wide-screen/narrow-screen design.

In the wide-screen/narrow-screen design, infants are presented with a basic design in which an object (e.g., a green ball) moves behind a screen. At the moment when one would expect the ball to reappear on the other side of the screen, a different-looking object (e.g., a red box) appears. The box stops at an outer location, rests for a brief period, and then returns behind the screen from which the ball reappears on the other side. This sequence continues until trial termination. Two different conditions are involved: a wide-screen condition, where the screen is of sufficient width to hide both objects at the same time (the expected condition) and a narrow-screen condition, where the screen is too narrow to hide both objects (the unexpected condition). The 
reasoning was that, if the infants, by means of the featural differences between the objects, would reason that they were indeed two different objects involved and subsequently draw the inference that the combined width of these two objects surpassed the width of the narrow screen, then it would result in a surprise reaction (i.e., longer looks) relative to the infants in the wide-screen condition. That was indeed what the results showed. The 9.5-month-old infants in the narrowscreen condition looked reliably longer than their equal-aged peers in the wide-screen condition [4, Exp. 3]. Subsequently, the results were replicated with 7.5- ([4, Exp. 4] and [5]) and 4.5-month-old infants [5]. Given the results provided from this series of experiments, even young infants may indeed be able to individuate objects by featural means.

However, there is still a debate over how the results from the wide-screen/narrow-screen design should be interpreted [6]. In contrast to the interpretation held by Needham and Baillargeon and their collaborators [7], Xu and Carey [8], $\mathrm{Xu}$ [9], and $\mathrm{Xu}$ et al. [10] have argued that infants may interpret the basic ball-box narrow screen event as a single object, which has changed its appearance. (Baillargeon [11] has recently described the box-ball narrow screen event in a "change violation" framework, rather than in an object individuation framework. Since our reasons for conducting the experiment were based on the original individuation terminology which is still widely accepted in the infant literature, we have maintained this terminology throughout the paper.)

Although a few recent studies [12, 13] have attempted to clarify how to interpret the wide-screen/narrow-screen design, the core of the dispute still remains. At a glance it may seem trivial whether science will ultimately settle on one interpretation or the other, but it is not: first, the two different interpretations offered result in markedly different developmental paths with respect to infants' use of different sources of information when attempting to individuate objects. Second, our understanding of object individuation has a substantial impact on several related fields of research. For instance, in order to learn new words in what Brown [14] called "the original word game", object individuation serves as a prerequisite in order for the infant to decide exactly to which object the adult is referring. Thus, because object individuation can be seen as a basic and important "general purpose tool" for human beings [2], it is important how we understand the developmental path of this issue.

We decided to replicate the original wide-screen/narrowscreen design with infants by using two different methodologies simultaneously: (a) the standard looking time paradigm (SLTP) and (b) eye tracking. The SLTP has, since it was developed in the mid 1980s, proven incredibly useful when conducting research with young infants. When applied to the wide-screen/narrow-screen design, the SLTP tells us how much the infants will attend to the "scoring field" in the different conditions (see Figure 1). However, the SLTP provides no information about what is happening within the scoring field. In order to shed further light on the dispute regarding whether infants actually parse the narrow screen condition as involving two different objects or a single object changing appearance, we might be especially interested in exactly such

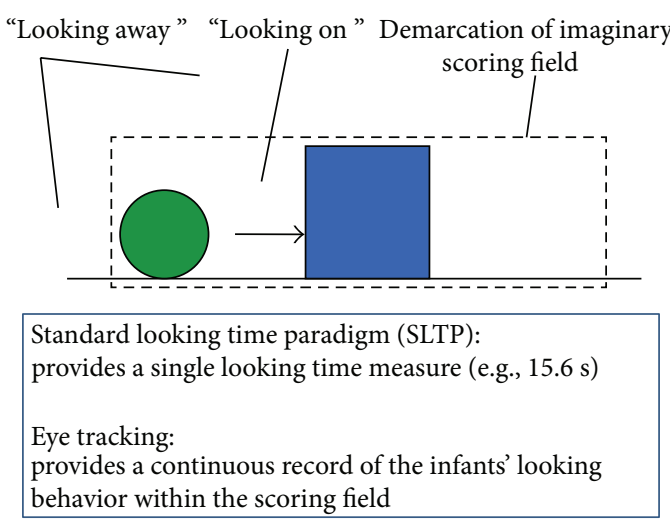

FIGURE 1: Schematic representation of output measures derived from the standard looking time paradigm (SLTP) and eye tracking, respectively, in the Narrow-Screen condition.

information. An increasing number of infant studies have recently used eye tracking methodology, but these studies have used eye tracking only (e.g., $[15,16])$, making it difficult to assess how the results obtained through eye tracking correspond to results obtained in the SLTP. In the present study we therefore employed both methodologies. By using the results from the SLTP as "baseline," but accompanied by eye tracking data, we had the opportunity to investigate what Aslin $[17,18]$ has phrased the microstructure of infants' visual attention, while maintaining the possibility of comparing the results of the two methodologies.

Both Wilcox and colleagues $[4,19]$ and $\mathrm{Xu}$ and collaborators $[10,20]$ have made use of adult ratings of reactions to the wide-screen/narrow-screen design. So far the results have been somewhat diverging-partly because the adults have been confronted with different questions across studies. Also, the sample sizes have been relatively small. Thus, so far no one has attempted to have large samples of adults choose between the two interpretations offered when presented with the narrow-screen scenario. In the present study we included such a study with a fairy large sample of adults.

\section{The Experiment}

Through eye tracking, we are able to test new hypotheses with regard to how to interpret the infants' looking behavior, from different theoretical perspectives: if the infants interpret the narrow-screen design as involving two distinct objects, as Wilcox and collaborators suggest, then we expect the infants in the Narrow-Test condition (with different objects) to look relatively longer at the screen (in order to search for the other object) compared to infants in the Narrow-Control condition (with identical objects), whereas this should not be expected if the infants parsed the narrow-screen event as suggested by $\mathrm{Xu}$ and Carey [8] (one object changing appearance). For the sake of convenience, we dub these hypotheses the screen hypotheses.

In our attempt to replicate the design used in the original study by Wilcox and Baillargeon [4, Exp. 3], we used the following four conditions (see Table 2). 
Condition 1 (Wide-Test) and 3 (Narrow-Test) were exactly the same conditions as those used in the original experiment by Wilcox and Baillargeon [4, Exp. 3]. Condition 2 (NarrowControl) was added in order to have a condition controlling for the possibility that the infants did not use featural information to discriminate between the objects. In condition 4 (Very-Narrow-Test) we used an even smaller screen (width $=17 \mathrm{~cm}$ ). The reason for running the Very-Narrow-Test condition was based on previous debate regarding whether the $21 \mathrm{~cm}$ narrow screen would actually be judged by participants as being sufficiently wide to simultaneously hide both the ball $(10.25 \mathrm{~cm})$ and the box $(11.75 \mathrm{~cm})$, given their combined width of $22 \mathrm{~cm}$ (cf. $[7,8]$ ).

We also decided to show a filmed and looped version of the Narrow-Test condition to a large sample of adults, in order, for the first time, to have an adult sample choose between the two opposing interpretations offered in the literature. This specific experiment is reported under a single heading in the results section.

\section{Method}

3.1. Participants. A total of 80 infants, 9.5 months of age ( 35 girls, mean age of 9 months, 17 days; range of 9 months, 8 days to 10 months, 0 days), were randomly assigned to one of four conditions (Wide-Test: $n=20$ [9 girls]; $M_{\text {age }}=9$ months, 15 days; range: 9 months, 8 days to 9 months, 21 days; Narrow-Control: $n=20$ [9 girls]; $M_{\text {age }}=9$ months, 18 days; range: 9 months, 12 days to 10 months, 0 days; Narrow-Test: $n=20$ [ 8 girls]; $M_{\text {age }}=9$ months, 16 days; range: 9 months, 8 days to 9 months, 29 days; Very-Narrow-Test: $n=20$ [9 girls]; $M_{\text {age }}=9$ months, 18 days; range: 9 months, 8 days to 9 months, 28 days).

Because we wanted to compare the SLTP data with the eye tracking data, we only included infants who could provide complete sets of both kinds of data. An additional 49 infants were tested but excluded from the analysis ( 15 due to infant fussiness, 12 due to mechanical errors, 15 due to missing [nine] or partly missing [six] eye tracking data, and seven due to imprecise eye tracking data). (Partly missing eye tracking data refers to trials where we obtained less than $50 \%$ valid gaze sample points in Tobii Studio. Imprecise data refers to trials where the tracking was clearly off target (e.g., clearly looking at the ball, while the tracking indicated that the infant looked at the screen). All exclusions were carried out prior to data analysis.) The infants were recruited through access to a birth register. All participating infants were full term and healthy. Besides receiving a teddy bear, no compensation was offered.

3.2. Apparatus. The reported experiment was a close replication of that reported in Wilcox and Baillargeon [4, Exp. 3]. The staged events took place in a large puppet theatre with a stage opening that was $94 \mathrm{~cm}$ wide and $41 \mathrm{~cm}$ high. The stage opening could be covered by a white curtain administered from the back of the theatre. We used two test objects: the ball was $10.25 \mathrm{~cm}$ in diameter, made of polystyrene, and painted green. The box was a $11.75 \mathrm{~cm}$ square, made of polystyrene, and painted red. The screen used in the familiarization trials was $45 \mathrm{~cm}$ wide and $20 \mathrm{~cm}$ high, made of plastic, and covered with yellow contact paper. The screen used in the Wide-Test condition was $30 \mathrm{~cm}$ wide and $20 \mathrm{~cm}$ high, made of plastic, and covered with blue contact paper. The screen used in the Narrow-Test/Control conditions was $21 \mathrm{~cm}$ wide and $20 \mathrm{~cm}$ high, made of plastic, and covered with blue contact paper. Thus, the objects and screens used were identical to those used in the original experiment with regard to shape, size, and color (except that our green ball had no dots and our red box had no silver stars). A few aspects of our setup were different, though.

First, we added the Very-Narrow-Test condition using an even smaller screen (width $=17 \mathrm{~cm}$ ) than the $21 \mathrm{~cm}$ screen (but with the same color and material) from the NarrowControl and Narrow-Test conditions. Second, instead of moving the objects by hand as in the reference study, we employed a custom made, computer controlled mechanical system using step motors. By having the object movements mechanized and computer-controlled, we increased the likelihood that all trials were equivalent for each infant, as well as across infants and conditions. Third, the unexpected event was not carried out by using a hollow box that could contain and hide the ball, as in the reference experiment. Instead, we used a single carrier, with one (visible) object above the stage floor and a second (invisible) object below the stage floor. Object changes were carried out by having the two objects (one above and one below the stage floor) switch positions while the carrier was occluded by the screen. The object switch took place in a $21 \mathrm{~cm}$ wide and $37 \mathrm{~cm}$ long opening in the stage floor, by having the carrier conduct a fast $180^{\circ}$ turn (orthogonal to the horizontal object movement path) by means of an additional step motor. This procedure was used regardless of condition. In the three test conditions (condition 1, 3, and 4) the objects were different at the two ends of the rotating carrier, and in the Narrow-Control condition the objects were identical. In that way, the noise from the additional step motor conducting the $180^{\circ}$ rotation was present in every single trial and thus gave the infants no cues about the present condition. Fourth, looking time scoring (SLTP) was not conducted through peepholes on either side of the theatre, as in the reference study, but via the output of a JVC hard disk camcorder (Model: GZ-MG255), mounted on axis in a small opening just below the stage.

Fifth, besides SLTP, we used eye tracking. In order to inform the eye tracker about what the infants saw, a digital SONY scene camera (Model: DCR-HC37E) was mounted on axis just above and slightly behind the infants' heads. Recording of eye movements was obtained using a Tobii X120 Eye Tracker mounted just below the upper edge of the stage floor. The X120 Eye Tracker is a free-standing model that needs no chin rest, head mount, or equivalent for the infants.

The infants sat in an autochair. The distance from the eyes of the infants to the staged objects was app. $83 \mathrm{~cm}$, while the distance from the eyes of the infants to the eye tracker was $70 \mathrm{~cm}$. The eyes of the infants were approximately 5 degrees above the stage floor. The mothers were sitting next to the infants, with their back towards the stage opening. 
3.3. Procedure. Prior to the experiment, each infant was calibrated using the manual 5 point calibration procedure in Tobii Studio. The calibration was conducted at screen position, by means of a thin board made of plastic, measuring $60 \mathrm{~cm}$ wide and $48 \mathrm{~cm}$ high. The fixed calibration points were lit up by a small LED light from the back of the panel. Immediately after the calibration, the experiment began. The procedure closely followed the reference study. Thus, each infant was presented with two familiarization trials, two pretest trials, and then, finally, four test trials. Each trial termination was accompanied by curtain fall.

3.3.1. The Familiarization Trials. The purpose of the familiarization trials was to make the infants familiar with the basic object movements and sounds involved in the experiment. As in the reference experiment, each infant saw two familiarization trials. Each trial began with the target object resting at the most extreme left position, with the center of the object positioned $24 \mathrm{~cm}$ left to the center of the stage. For the infants in all conditions other than the Narrow-Control condition with the box, the target object was always the ball; for the infants in the Narrow-Control condition with the box, the target object was always the box. (In the remainder of the procedure description for the familiarization trials, we describe the event as if the ball was the target object.) From the infants' perspective, the majority of the remaining parts of the stage floor were covered by the $45 \mathrm{~cm}$ wide familiarization screen. When the infant had looked at the ball for 2 consecutive seconds, it began moving towards the center and behind the screen, with a stable velocity of app. $12 \mathrm{~cm} / \mathrm{sec}$. While the ball was hidden behind the screen, the mechanical setup conducted the $180^{\circ}$ object rotation described above, making the object mounted at the other end of the rotating carrier appear on the stage floor, before it moved to the most extreme right position $(24 \mathrm{~cm}$ to the right of the center of the stage), where it rested for $1 \mathrm{sec}$. Subsequently, the trajectory was reversed (including another rotation, making the ball reappear on the stage floor) until the ball came to rest at the initial position to the far left of the center. This sequence continued until trial termination. Note that, because of the wide screen, the rotation and the eventual second object mounted at the other end of the rotating carrier were not visible to the infants during the familiarization trials, but the procedure was maintained throughout the familiarization trials anyway, in order to preserve the timing of the later experiment trials, as well as the sounds that would be coming from the theatre. Each trial ended when the infant had looked away for 2 consecutive seconds, after having looked at the event for 10 cumulative seconds or if the infant had looked at the scenario for 60 cumulative seconds without having looked away for 2 consecutive seconds.

In summary, the materials used, the procedure administered, and the trial termination criteria for the familiarization trials were very similar to those employed in the reference experiment. Two pretest trials followed immediately after the familiarization trials.

3.3.2. The Pretest Trials. In the pretest trials, infants saw the stationary positions at each of the two outer positions
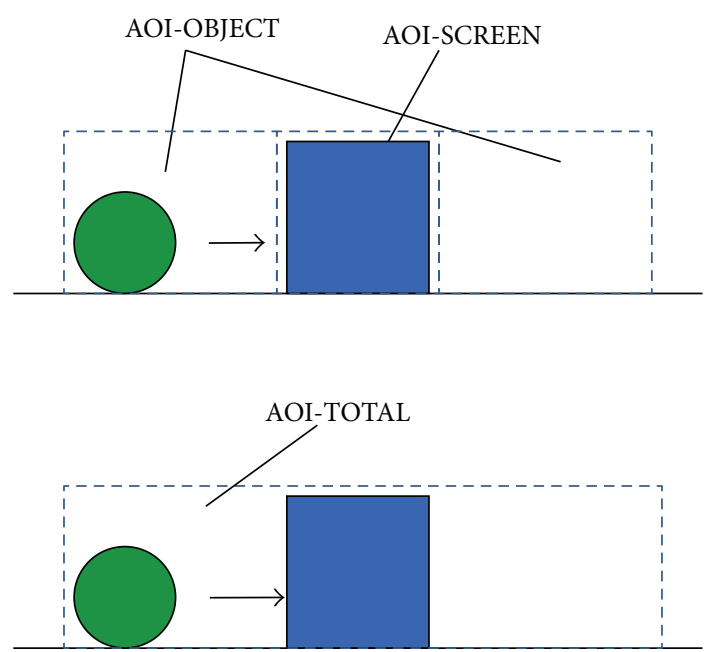

FIgURE 2: Schematic representation of the AOI's employed in the analysis. Note that the sizes of AOI-SCREEN and AOI-OBJECT vary depending on the size of the screen in the given condition.

(extreme left and extreme right of center, resp.), using the same objects and screens as in the subsequent test events. To illustrate, infants in the Wide-Test condition would, in the first pretest trial, see the green ball resting to the left with the wide screen located at the center of the stage, whereas the second pretest trial involved the red box resting to the right of the wide screen and so forth. The termination criteria were as follows: each trial ended when the infant had looked at the display for 2 consecutive seconds after having looked at the scenario for 5 cumulative seconds or if the infant looked the display for 30 cumulative seconds without having looked away for 2 consecutive seconds. (We were unable to always fulfill the minimum looking-on criteria $(5 \mathrm{sec})$ for the pretest trials for the second object. In case an infant, after having looked at the second object for some time, then looked away and became fussy, the pretest procedure was terminated by the experimenter, and we moved directly to the test trials. Later replications $[5,20]$ of the original design have left out the pretest sequence too, so we may assume that others have had similar problems.) The four test trials immediately followed the pretest trial.

3.3.3. Test Trials. The four test trials were analogous to the familiarization trials with three important exceptions: first, the screen used was not the $45 \mathrm{~cm}$ wide familiarization screen, but the screen relevant for the given test condition being conducted (see Figure 2). Second, and in complete accordance with the reference experiment (Wilcox and Baillargeon, 1998, page 121), the trial termination criteria were different: each trial ended when the infant had looked away for 0.5 consecutive seconds, after having looked at the scenario for 5 cumulative seconds or after having looked at the display for 60 cumulative seconds without having looked away for 0.5 consecutive seconds. Third, besides SLTP, the infants' eye movements were recorded in the four test trials. 
It is important to note that the technical initiation and termination criteria of the trials were exclusively dependent on the SLTP throughout the experiment-exactly as in the reference experiment. Eye tracking recording was only an add-on, and only eye tracking data from the four test trials were used in the data analysis.

Whereas the Wide-Test and the Narrow-Test conditions were completely identical to the conditions employed in the reference study, the Narrow-Control and Very-Narrow-Test conditions were different. In the Narrow-Control condition, half of the infants $(n=10)$ were tested with two identical exemplars of the green ball used in the other conditions, whereas the other half of the children $(n=10)$ were tested with two identical exemplars of the red box used in the other conditions. In the Very-Narrow-Test condition, the procedure was identical to that used in the Narrow-Test condition, except for the use of a very-narrow screen in the pretest and the test trials.

Eye tracking data were recorded and analyzed using Tobii Studio 1.3. On all trials, the Tobii Fixation filter was applied. The filter calculates fixations based on gaze coordinates that are within a region of 35 pixels $\left(1.5^{\circ}\right.$ visual angle in this study). The minimum possible fixation duration is $41.7 \mathrm{~ms}$. The total area of interest measured $60 \mathrm{~cm}$ (wide) $\times 20 \mathrm{~cm}$ (high), exactly encompassing the area constituted by the possible object movements and the screen(s). For all four test trials this total area of interest was divided into three areas of interest (AOI): one that covered the screen and, therefore, varied in size accordingly (AOI-SCREEN Wide-Test $_{\text {: }}$ $30 \mathrm{~cm}$ wide, $20 \mathrm{~cm}$ high, visual angle: $14.0^{\circ} \times 9.3^{\circ}$; AOISCREEN $_{\text {Narrow-Control and Narrow-Test }}: 21 \mathrm{~cm}$ wide, $20 \mathrm{~cm}$ high, visual angle: $9.8^{\circ} \times 9.3^{\circ}$; AOI-SCREEN Very-Narrow-Test $: 17 \mathrm{~cm}$ wide, $20 \mathrm{~cm}$ high, visual angle: $7.9^{\circ} \times 9.3^{\circ}$ ) and two equalsized AOI's that covered the left and right sides of the scene, which contained the objects that would be visible. Again, the size of these AOI's varied according to condition (AOI-OBJECT Wide-Test $: 15 \mathrm{~cm}$ wide, $20 \mathrm{~cm}$ high, visual angle: $7.0^{\circ} \times 9.3^{\circ} ;$ AOI-OBJECT ${ }_{\text {Narrow-Control and Narrow-Test }}$ : $19.5 \mathrm{~cm}$ wide, $20 \mathrm{~cm}$ high, visual angle: $9.1^{\circ} \times 9.3^{\circ}$; AOIOBJECT Very-Narrow-Test $: 21.5 \mathrm{~cm}$ wide, $20 \mathrm{~cm}$ high, visual angle: $\left.10.0^{\circ} \times 9.3^{\circ}\right)$. Finally, we used one big AOI-TOTAL, encompassing the total scoring field $(60 \mathrm{~cm}$ wide, $20 \mathrm{~cm}$ high, visual angle: $27.9^{\circ} \times 9.3^{\circ}$ ) and thus equivalent to the "looking on" field when using the SLTP (see Figure 2).

\section{Results}

We first analyzed the data from the SLTP in order to ascertain whether we replicated the results from the reference study [4]. In our analysis, we followed the analytic strategy of the reference experiment.

4.1. SLTP Data. The infants' looking times from the two familiarization trials were averaged and analyzed by means of a one-way ANOVA, with condition (Wide-Test versus Narrow-Control versus Narrow-Test versus Very-Narrow Test) as a between-subjects factor. The analysis revealed no significant main effect, $F(3,76)=2.53, P=0.06, \eta_{p}^{2}=0.09$,

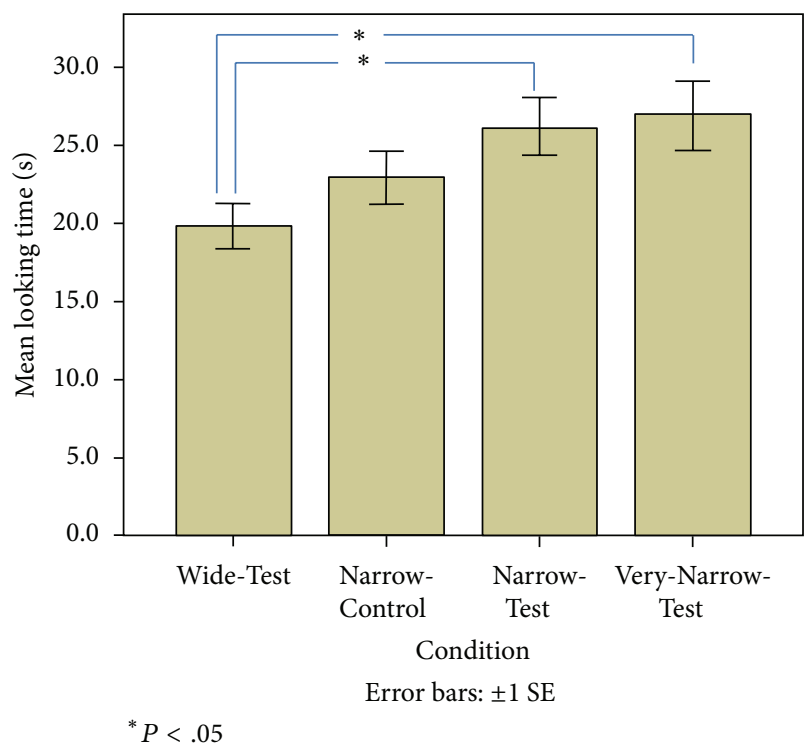

FIGURE 3: Mean looking times in the four conditions, using the standard looking time paradigm.

although there was a tendency for the infants in the VeryNarrow-Test condition to look longer at the familiarization trials $\left(M_{\text {Very-Narrow-Test }}=37.6 \mathrm{~s}, \mathrm{SD}_{\text {Very-Narrow-Test }}=12.4\right)$ than the infants in the other conditions $\left(M_{\text {Wide-Test }}=27.3 \mathrm{~s}\right.$, $\mathrm{SD}_{\text {Wide-Test }}=9.4 ; M_{\text {Narrow-Control }}=30.4 \mathrm{~s} ; \mathrm{SD}_{\text {Narrow-Control }}=$ $\left.12.3 ; M_{\text {Narrow-Test }}=31.0 \mathrm{~s}, \mathrm{SD}_{\text {Narrow-Test }}=14.2\right)$. Since we were unable to fulfill the minimum pretest criteria (see footnote three), no analyses of these data are reported.

The infants' looking times for the four test trials were averaged and analyzed by means of a one-way ANOVA, with condition (Wide-Test versus Narrow-Control versus NarrowTest versus Very-Narrow-Test) as between-subjects factor. The results revealed a main effect of condition, $F(3,76)=$ 3.06, $P=0.03, \eta_{p}^{2}=0.11$, indicating that the looking times in the test trials varied significantly across conditions $\left(M_{\text {Wide-Test }}=19.8 \mathrm{~s}, \mathrm{SD}_{\text {Wide-Test }}=6.5 ; M_{\text {Narrow-Control }}=22.9 \mathrm{~s}\right.$; $\mathrm{SD}_{\text {Narrow-Control }}=7.8 ; M_{\text {Narrow-Test }}=26.2 \mathrm{~s}, \mathrm{SD}_{\text {Narrow-Test }}=$ $8.4 ; M_{\text {Very-Narrow-Test }}=26.9 \mathrm{~s}, \mathrm{SD}_{\text {Very-Narrow-Test }}=10.2$; see Figure 3).

Following the analytic strategy from [4, Exp. 3], we conducted a planned comparison between the Wide-Test condition and the Narrow-Test condition by means of a oneway ANOVA, with the two conditions as between-subjects factor. The results revealed a simple effect of condition, $F(1,38)=7.20, P=0.01, \eta_{p}^{2}=0.16$, replicating a main result of the reference study. Thus, the infants in the NarrowTest condition did indeed look longer than their peers in the Wide-Test condition.

Another planned comparison between the Narrow-Test condition and the Narrow-Control condition, conducted by means of a one-way ANOVA with the two conditions as between-subjects factor, did not produce a reliable simple effect, $F(1,38)=1.62, P=0.21, \eta_{p}^{2}=0.04$ $\left(M_{\text {Narrow-Test }}=26.2 \mathrm{~s} ; \mathrm{SD}_{\text {Narrow-Test }}=8.4 ; M_{\text {Narrow-Control }}=\right.$ 
$22.9 \mathrm{~s}, \mathrm{SD}_{\text {Narrow-Control }}=7.8$ ). This negative result was underscored by the fact that, within the Narrow-Control condition, the looking times for the 10 infants seeing two (identical-looking) green balls were clearly not significantly different from the looking times for the remaining 10 infants in the same condition, who saw two (identical-looking) red boxes, $F(1,18)=0.016 ; M_{\text {Ball-Ball }}=23.1 \mathrm{~s}, \mathrm{SD}_{\text {Ball-Ball }}=8.9$; $M_{\text {Box-Box }}=22.7 \mathrm{~s}, \mathrm{SD}_{\text {Box-Box }}=7.0$. Note that being unable to empirically support the claim that the infants actually used featural information to discriminate between the two objects involved in the test conditions is nontrivial because the logic behind the wide-screen/narrow-screen design is based on the assumption that infants do indeed use the available featural information to discriminate between the objects involved (cf. [4, page 118]).

Two additional comparisons were made in order to see how the infants faired in the Very-Narrow-Test condition. First, a one-way ANOVA with the Wide-Test condition and the Very-Narrow-Test condition as between-subjects factor and with mean looking time as dependent variable revealed a simple significant effect of condition $(F[1,38]=6.88, P=$ $\left.0.01, \eta_{p}^{2}=0.15\right)$. The infants in the Very-Narrow-Test condition obtained reliably longer looking times relative to their peers in the Wide-Test condition $\left(M_{\text {Very-Narrow-Test }}=26.9 \mathrm{~s}\right.$; $\mathrm{SD}_{\text {Very-Narrow-Test }}=10.2 ; M_{\text {Wide-Test }}=19.8 \mathrm{~s} ; \mathrm{SD}_{\text {Wide-Test }}=$ 6.5). Second, a one-way ANOVA with the Very-Narrow-Test condition and the Narrow-Test condition as between-subjects factor did not result in a reliable simple effect, $F(1,38)=$ $0.05, P=0.82, \eta_{p}^{2}=0.001\left(M_{\text {Very-Narrow-Test }}=26.9 \mathrm{~s}\right.$; $\mathrm{SD}_{\text {Very-Narrow-Test }}=10.2 ; M_{\text {Narrow-Test }}=26.2 \mathrm{~s} ; \mathrm{SD}_{\text {Narrow-Test }}=$ 8.4). These additional comparisons were not part of the study we set out to replicate because Wilcox and Baillargeon [4] did not at that time include a Very-Narrow-Test condition. However, the results obtained in the present study from these additional comparisons are nevertheless in accordance with the rationale behind the original study. Based on the reasoning from Wilcox and Baillargeon [4, Exp. 3], the infants in the Very-Narrow-Test condition should fair exactly like their peers in the Narrow-Test condition. The reason is that the quantitative difference between the widths of the two screens should make no qualitative difference with regard to looking time as long as the width of each screen is smaller than the combined width of the two different objects involved. And this was exactly what the results showed.

4.2. Eye Tracking Data. In order to verify to what extent data obtained through eye tracking methodology corresponds to the data obtained through the SLTP, we initially ran, for each participant, a bivariate correlation between the mean total fixations, AOI-TOTAL (all AOI's summed), as measured by the eye tracking procedure, and the mean (test) looking times obtained through the SLTP. We ran this correlation across all four conditions. The results yielded a very strong correlation, $r=0.94, P<0.0001$, indicating that the two methodologies do indeed, to a very large extent, tap into the same aspects of infant looking behavior.

Subsequently, we again employed the analytic strategy from the reference study but now focused on the eye

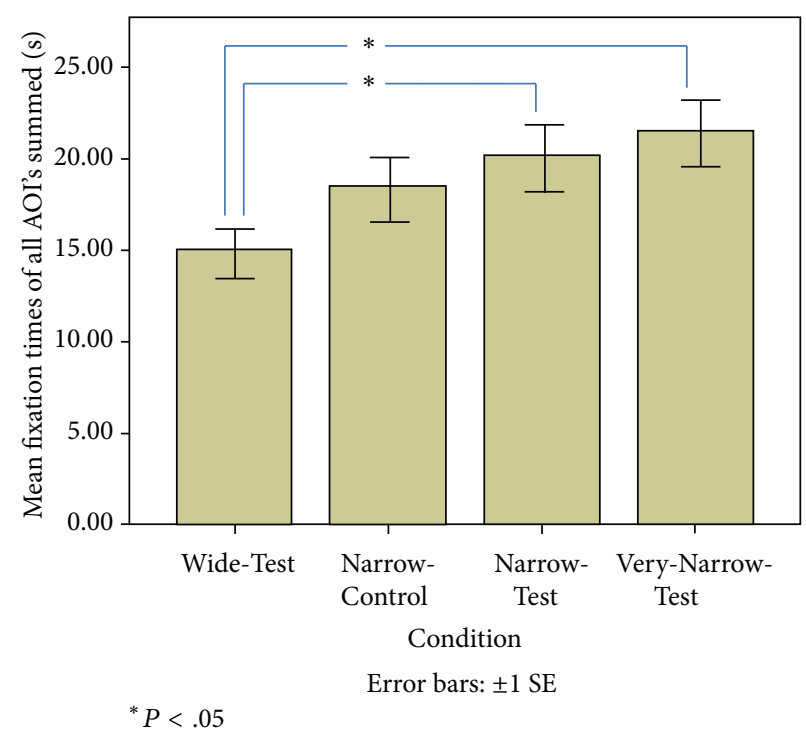

FIgURE 4: Mean fixation times in all AOI's summed (AOI-TOTAL).

TABLE 1: Mean eye tracking fixation times in AOI's across the four conditions.

\begin{tabular}{lcccc}
\hline \multirow{2}{*}{ Condition } & \multicolumn{2}{c}{ AOI-OBJECT (in sec) } & \multicolumn{2}{c}{ AOI-SCREEN (in sec) } \\
& $\mathrm{M}_{\text {AOI-OBJ }}$ & $\mathrm{SD}_{\text {AOI-OBJ }}$ & $\mathrm{M}_{\text {AOI-SCREEN }}$ & SD $_{\text {AOI-SCREEN }}$ \\
\hline Wide-Test & 10.86 & 4.58 & 4.14 & 2.52 \\
$\begin{array}{l}\text { Narrow- } \\
\text { Control }\end{array}$ & 14.57 & 6.45 & 3.92 & 2.14 \\
$\begin{array}{l}\text { Narrow- } \\
\text { Test }\end{array}$ & 15.91 & 6.52 & 4.24 & 2.20 \\
$\begin{array}{l}\text { Very- } \\
\text { Narrow- }\end{array}$ & 17.90 & 6.79 & 3.59 & 2.00 \\
Test & & & & \\
\hline
\end{tabular}

tracking data. Thus, the infants' mean total fixations, AOITOTAL (all AOI's summed), for the four test trials were analyzed by means of a one-way ANOVA, with condition (Wide-Test versus Narrow-Control versus Narrow-Test versus Very-Narrow-Test) as a between-subjects factor. The results revealed a main effect of condition, $F(3,76)=$ 2.77, $P=0.048, \eta_{p}^{2}=0.10$, indicating that the looking times of AOI-TOTAL in the test trials varied significantly across conditions $\left(M_{\text {Wide-Test }}=15.0 \mathrm{~s}, \mathrm{SD}_{\text {Wide-Test }}=6.0\right.$; $M_{\text {Narrow-Control }}=18.5 \mathrm{~s} ; \mathrm{SD}_{\text {Narrow-Control }}=7.8 ; M_{\text {Narrow-Test }}=$ $20.2 \mathrm{~s}, \mathrm{SD}_{\text {Narrow-Test }}=8.2 ; M_{\text {Very-Narrow-Test }}=21.5 \mathrm{~s}$, $\mathrm{SD}_{\text {Very-Narrow-Test }}=7.9$; see Figure 4 ).

We again conducted a planned comparison between the Wide-Test condition and the Narrow-Test condition by means of a one-way ANOVA, with the two conditions as between-subjects factor and AOI-TOTAL as dependent variable. This analysis revealed a significant simple effect of condition $\left(F[1,38]=5.13, P=0.03, \eta_{p}^{2}=0.12\right)$. Thus, we again replicated a basic finding from the reference study that infants do look longer at the Narrow-Test condition relative to the Wide-Test condition, but this time by means of eye tracking data. 
TABLE 2: Overview of conditions.

\begin{tabular}{lcccc}
\hline No. & Description & Abbreviation & $N$ & Age in months \\
\hline 1 & Wide screen, different objects (ball-box) & Wide-Test & 20 & 9.5 \\
2 & Narrow-screen, same objects (ball-ball or box-box) & Narrow-Control & 20 & 9.5 \\
3 & Narrow screen, different objects (ball-box) & Narrow-Test & 20 & 9.5 \\
4 & Very narrow screen, different objects (ball-box) & Very-Narrow-Test & 20 & 9.5 \\
\hline
\end{tabular}

The second planned comparison between the NarrowTest condition and the Narrow-Control condition did not, however, result in a significant effect $(F[1,38]=0.43, P=$ $\left.0.52, \eta_{p}^{2}=0.01\right)$. Resembling the results obtained through the SLTP, the eye tracking data failed to provide evidence that the infants used featural information to discriminate between the objects involved. For the infants participating in the NarrowControl condition, we again decided to conduct a comparison between the 10 infants who saw two identical green balls and the remaining 10 infants in the condition, who saw two identical red boxes. We did so by means of a one-way ANOVA with AOI-TOTAL as dependent variable. The results from this comparison were, again, insignificant $(F[1,18]=0.40$, $P=0.54, \eta_{p}^{2}=0.02 ; M_{\text {Ball-Ball }}=19.6 \mathrm{~s}, \mathrm{SD}_{\text {Ball-Ball }}=8.6$; $M_{\text {Box-Box }}=17.4 \mathrm{~s}, \mathrm{SD}_{\text {Box-Box }}=7.3$ ).

We then exploited the possibility, provided by the eye tracking data, to break down the looking behavior within the "scoring field" into AOI's, as previously described (cf. Table 2 and Figure 3). Thus, the infants' eye tracking data for the four test trials were averaged and analyzed by means of a one-way ANOVA with condition (Wide-Test versus Narrow-Control versus Narrow-Test versus Very-Narrow-Test) as betweensubjects factor and with AOI-OBJECT and AOI-SCREEN as dependent variables. The results revealed a main effect of condition on AOI-OBJECT, $F(3,76)=4.65, P=0.005, \eta_{p}^{2}=$ 0.16 , whereas condition had no impact on AOI-SCREEN, $F(3,76)=0.34, P=0.80, \eta_{p}^{2}=0.01$ (see Figure 5).

Thus, while the mean OBJECT fixations varied across conditions, the mean SCREEN fixations clearly did not (see Table 1 for Means).

We again conducted two planned contrasts: a one-way ANOVA with the Wide-Test condition and the Narrow-Test condition as between-subjects factor, and AOI-OBJECT as dependent variable yielded (again) a significant simple effect $\left(F[1,38]=8.03, P=0.007, \eta_{p}^{2}=0.17\right.$; see Table 1 for Means and SD's), whereas the other planned comparison, with the Narrow-Test condition and the Narrow-Control condition as between-subjects factor and AOI-OBJECT as dependent variable, was insignificant $(F[1,38]=0.43$, $\left.P=0.52, \eta_{p}^{2}=0.01\right)$. By means of a one-way ANOVA, we conducted a comparison between the 10 participants in the Narrow-Control condition who saw two identical balls and the remaining 10 infants from the same condition, who saw two identical boxes, with the AOI-OBJECT fixations as dependent variable. There was no significant difference between these two subgroups of the Narrow-Control condition, $F(1,18)=0.78, P=0.39, \eta_{p}^{2}=0.05$. Thus, once again, there was no indication in the data that the infants used

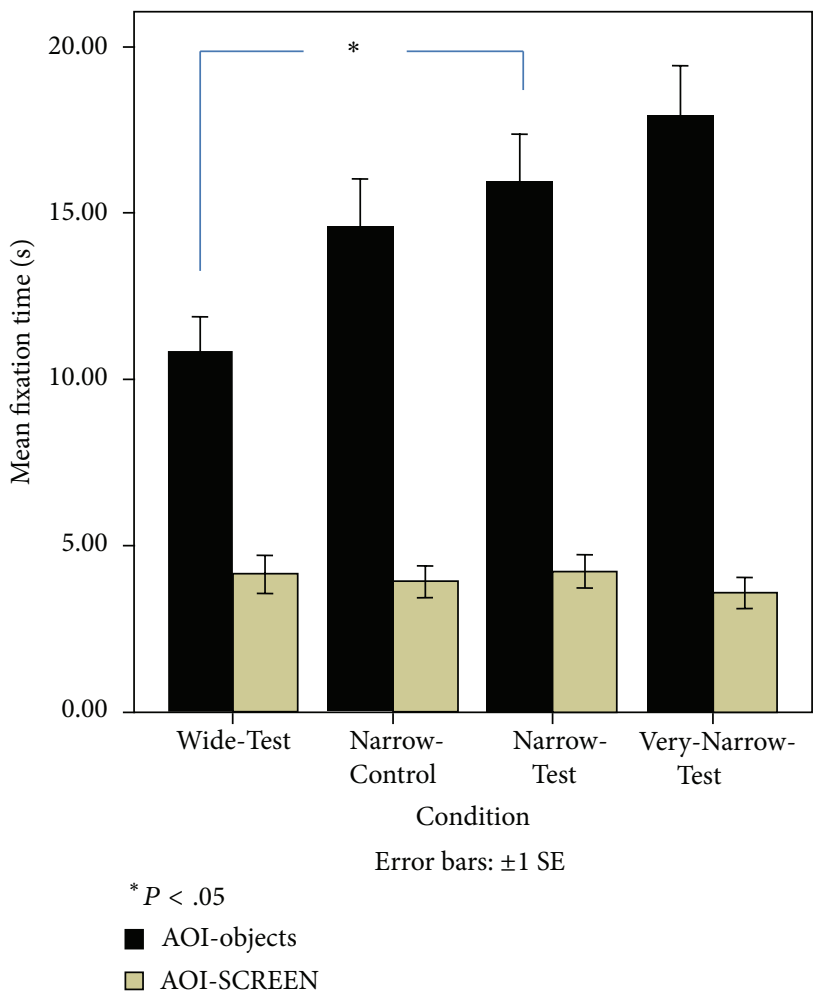

FIgURE 5: Mean fixation time in AOI's across the four conditions.

the available featural information in order to discriminate between the green ball $\left(M_{\text {Ball-Ball }}=15.8 \mathrm{~s}, \mathrm{SD}_{\text {Ball-Ball }}=6.6\right)$ and the red box $\left(M_{\text {Box-Box }}=13.3 \mathrm{~s}, \mathrm{SD}_{\text {Box-Box }}=6.3\right)$.

In order to test our screen hypotheses, with regard to the looking time devoted to the screens in the NarrowTest/Control conditions, we conducted a planned comparison by means of a one-way ANOVA, with condition (NarrowControl versus Narrow-Test) as a between-subjects factor and AOI-SCREEN fixations as dependent variable. As is already clear from Figure 6 and Table 1, no main effect was present, $F(1,38)=0.21, P=0.65, \eta_{p}^{2}=0.006$.

Provided our screen hypotheses are valid, then our results seem to support the interpretation held by $\mathrm{Xu}$ and Carey [8]. However, as will be clear, our screen hypotheses may not be the most obvious choice when interpreting the data.

Analogous to the additional comparisons conducted with the SLTP data in order to see how the infants faired in the Very-Narrow-Test condition, we replicated the two ANOVAs, 
but this time based on eye tracking data. First, a oneway ANOVA with the Wide-Test condition and the VeryNarrow-Test condition as between-subjects factor and with the infants' mean total fixations, AOI-TOTAL, as dependent variable, revealed a simple significant effect of condition $\left(F[1,38]=8.55, P=0.006, \eta_{p}^{2}=0.18\right)$. The infants in the Very-Narrow-Test condition obtained reliably longer looking times relative to their peers in the Wide-Test condition $\left(M_{\text {Very-Narrow-Test }}=21.5 \mathrm{~s} ; \mathrm{SD}_{\text {Very-Narrow-Test }}=7.9\right.$; $\left.M_{\text {Wide-Test }}=15.0 \mathrm{~s} ; \mathrm{SD}_{\text {Wide-Test }}=6.0\right)$. Second, a oneway ANOVA with the Very-Narrow-Test condition and the Narrow-Test condition as between-subjects factor did not result in a reliable simple effect, $F(1,38)=0.28, P=0.60$, $\eta_{p}^{2}=0.007\left(M_{\text {Very-Narrow-Test }}=21.5 \mathrm{~s} ; \mathrm{SD}_{\text {Very-Narrow-Test }}=\right.$ 7.9; $M_{\text {Narrow-Test }}=20.2 \mathrm{~s} ; \mathrm{SD}_{\text {Narrow-Test }}=8.2$ ). The results from these additional comparisons using eye tracking data are completely equivalent to the results from the additional analysis obtained by the SLTP as well as being in accordance with the rationale behind the original study by Wilcox and Baillargeon [4, Exp. 3].

4.3. Adult Participants, Procedure, and Results. A group of 147 of adults $\left(M_{\text {age }}=22.1\right.$ years, $\mathrm{SD}_{\text {age }}=4.3$, range $=19$ 45; 23 males) participated in the study, presented under the heading: "assessment of a perceptual event". All participants were 1st semester psychology students who, at that point, had had no courses in developmental/cognitive psychology and thus were presumably naive with regard to the widescreen/narrow-screen design.

Seated in a large auditorium, the participants were shown a filmed and looped sequence (duration $=3 \mathrm{~min}$.) of the Narrow-Test event, that is, the ball-box sequence with a narrow screen (width $=21 \mathrm{~cm}$ ). Meanwhile, the participants were asked to tick the option on their instruction sheet that, in their opinion, best answered the following question, Q1: "Is there anything unusual about the event?" The five response categories were (1) "Yes, the screen is definitely too small to simultaneously hide the ball and the box", (2) "The screen is probably too small to simultaneously hide the ball and the box", (3) "Maybe", (4) "The event is probably not unusual in any respect", and (5) "No, the event is definitely not unusual in any respect." When the participants had ticked their answer, they turned the page on the instruction sheet. Here, it was clearly stated that the second question should only be answered if they had thought the event was unusual to some extent; that is, if as their response to Q1, they had ticked either (1), (2), or (3). The participants were then instructed to respond by choosing between two descriptions of the event and were instructed to read through both descriptions before they made their decision. The two descriptions to choose from were as follows.

(A) "It looks as if the event contains a single object that remarkably changes appearance while the object is hidden for a moment behind the screen."

(B) "It looks as if the event contains two objects, and that the unusual aspect is that both objects-considering the size of the objects relative to the width of the

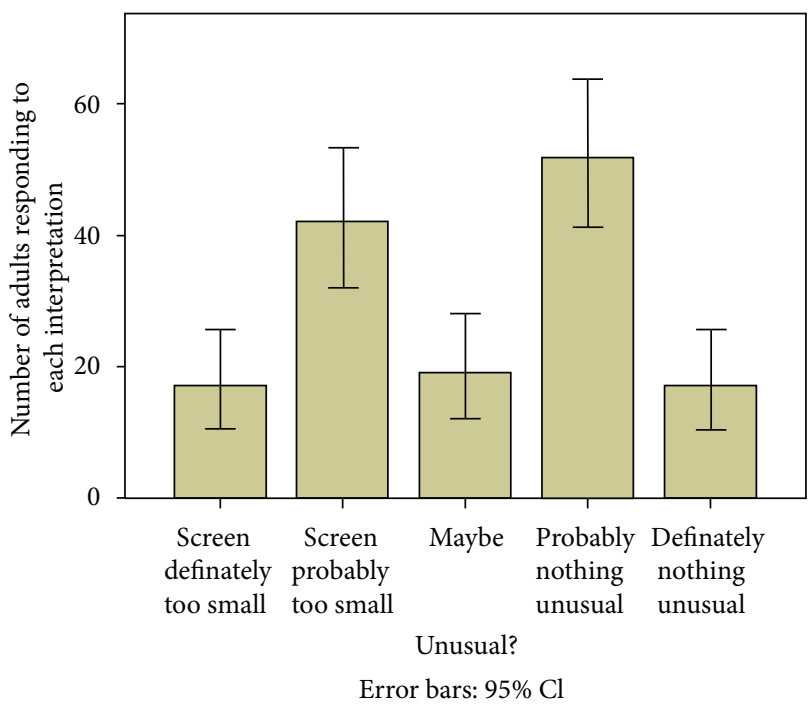

FIGURE 6: Schematic representation of adults' evaluations of the unusualness of the Narrow-Test event.

screen - cannot be hidden simultaneously behind the screen."

The order of the presentation of the two different descriptions was balanced across participants.

The results from the responses to Q1 revealed that the participants did not agree about the unusualness of the Narrow-Test event (see Figure 6). The two most frequently chosen ratings were (2) "The screen is probably too small to simultaneously hide the ball and the box" (28.6\%) and (4) "The event is probably not unusual in any respect" (35.4\%). This result indicates that the Narrow-Test event, at least among adults, may not be as unambiguously "unusual" or "unexpected" as the originators of the task might suspect.

Out of the 147 participants, 78 participants had ticked either (1), (2), or (3) and thus were qualified for the forcedchoice task of choosing between the two proffered interpretations. Of the 78 participants, two subjects had not ticked any of the proffered interpretations, so only 76 respondents were included in the analysis. The results revealed that the participants were completely divided between the two interpretations since exactly half of the participants $(n=$ 38) chose interpretation (A), whereas the other half $(n=$ 38) ticked interpretation (B). Thus, adults did not seem to agree about the unusualness of the Narrow-Test event, and among those who did find the event unusual to at least some extent, there were no consensus at all with regard to which interpretation to choose.

4.4. Additional Analysis of Infants. At face value, the data from the infants seem to support the interpretation offered by $\mathrm{Xu}$ and Carey [8]. However, $\mathrm{Xu}$ and Carey's interpretation relies on the assumption that the infants do detect a change in appearance [8, page 288], and there is no evidence in the present experiment to suggest that the infants used the available featural information to discriminate between the objects. In order to follow this suspicion further, we 
performed additional analyses. In the reference experiment, and hence also in our experiment, the green ball is invariably present on the left side of the screen, whereas the red box is always located on the right side in all test conditions (all conditions except the Narrow-Control condition). We exploited this feature by pooling together the eye tracking fixations on the left side of the screens (AOI-LEFT) with the eye tracking fixations on the right side of the screen (AOIRIGHT), across the three test conditions $(n=60)$, where the ball always appeared on the left side of the screen, whereas the box invariably appeared on the right. A simple paired sample $t$-test revealed that there was no difference, $t(59)=-1.55, P=$ $0.13, r=0.20$, between the AOI-LEFT $\left(M_{\text {Ball }}=7.2 \mathrm{~s}, \mathrm{SD}_{\text {Ball }}=\right.$ 3.4) and the AOI-RIGHT $\left(M_{\mathrm{Box}}=7.7 \mathrm{~s}, \mathrm{SD}_{\mathrm{Box}}=3.7\right)$. Thus, even across all conditions, there is no indication in the data that the infants used the available featural information to discriminate between the two different-looking objects involved in the three test conditions.

Because the two existing interpretations of results derived from previous studies using the wide-screen/narrow-screen design $[4,8]$ are both based on the assumption that the infants do discriminate between objects on the basis of featural information, we need to look for alternative interpretations in order to explain the present data. As evidenced in Figures $3-5$, there seems to be a trend that, as the "visual object field" increases (i.e., as narrower screens are used), looking times/fixation increase as well. The exact widths of the screens used in the present study were based on previous studies using variations of the same design. Given the cognitive rationale behind these designs, the screen widths reflect nominal and relational qualities ("Wide", "Narrow", and "VeryNarrow"), rather than absolute quantities. In order to obtain a more precise assessment of the relation between screen sizes and looking times, we computed a new continuous variable, the visual object field (VOF), which indicates the percentage of the width of the entire scoring field that was taken up by the width of the visual object field. We created this variable separately for each screen employed ([in each case, width of entire scoring field - screen width] $* 100 /$ width of entire scoring field). This new variable had three different values: $\mathrm{VOF}=50 \%(n=20$; Wide-Test $), \mathrm{VOF}=65 \%$ ( $n=40$; Narrow-Test and Narrow-Control combined), and $\mathrm{VOF}=72 \%(n=20$, Very-Narrow-Test $)$. We then computed three graphs displaying the relationship between VOF and looking time measures, based on the following data: (a) the relation between VOF and looking time from the SLTP (see Figure 7), (b) the relation between VOF and AOITOTAL from the eye tracking data (see Figure 8(a)), and (c) the relation between VOF and AOI-OBJECT from the eye tracking data (see Figure 8(b)). Whereas Figures 7 and 8(a) are equivalent, as they both display total looking time as a function of the size of the VOF, Figure 8(b) only displays the looking time devoted to the visual object field as a function of the size of the VOF. When it makes sense to display Figure 8(b) in relation to Figures 7 and 8(a), it is because the looking time devoted to the screens alone does not vary with screen size, as evidenced in Figure 5.

Based on the cognitive hypothesis held by Wilcox and Baillargeon [4], we should expect a qualitative shift in the

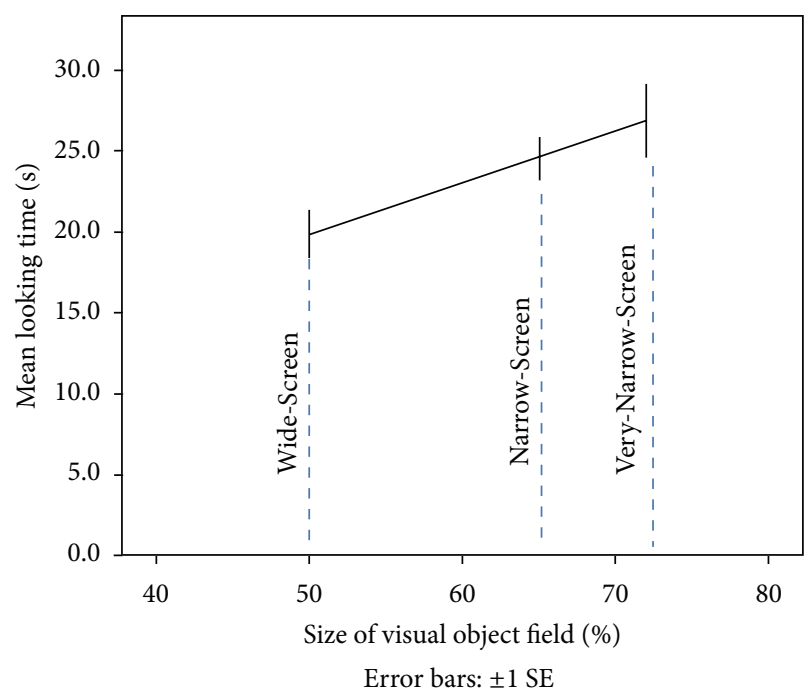

FIGURE 7: Graphic representation of the relation between size of the visual object field and Mean Looking Time from the standard looking time paradigm (SLTP).

relation between screen width and looking time. According to their rationale [4, page 118], the important feature is whether the screen belongs to the wide-screen or the narrow-screen category (relative to the combined widths of the objects involved), whereas the exact width of a given screen within a certain screen category should be less important. The results from the present study are not in accordance with these suppositions. Instead, and as indicated by Figures 7 and 8 , the results from the present study suggest that the relation between the Visual Object Field and the looking times may be linear, suggesting no qualitative shift. It should be noted that, to the best of our knowledge, no previous study using the wide-screen/narrow-screen design has used more than two different screen widths for their test trials. Consequently, in the existing literature, any potential linearity would have been impossible to detect.

In order to test for linearity in the data from the present study, we conducted three separate one-way ANOVAs, all with the size of the VOF as an independent variable (50\% VOF versus $65 \%$ VOF versus $72 \%$ VOF) and with either (a) mean looking time based on the SLTP (see Figure 7), (b) AOI-TOTAL from the eye tracking data (see Figure 8(a)), or (c) AOI-OBJECT from the eye tracking data (see Figure 8(b)) as dependent variables. All three ANOVAs revealed significant linear trends (SLTP as dependent variable (see Figure 7): $F[2,77]=7.58, P=0.007, \eta_{p}^{2}=0.10$; AOI-TOTAL from the eye tracking data as dependent variable (see Figure 8(a)): $F[2,77]=7.86, P=0.006, \eta_{p}^{2}=0.10$; AOI-OBJECT from the eye tracking data as dependent variable (see Figure $8(\mathrm{~b})$ ): $\left.F[2,77]=13.48, P=0.0004, \eta_{p}^{2}=0.18\right)$.

\section{Discussion}

The results from the present study are consistent and clear. By means of employing SLTP and eye tracking simultaneously, 


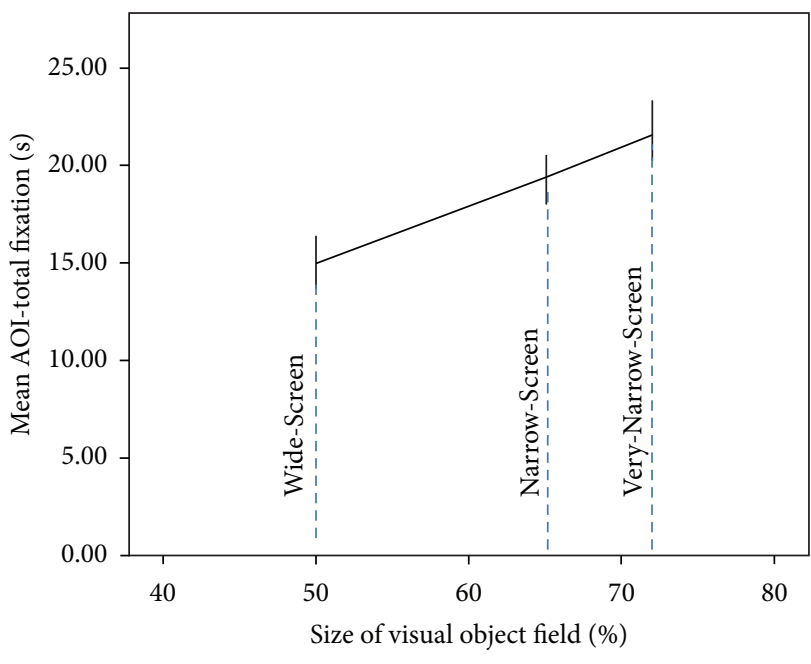

(a)

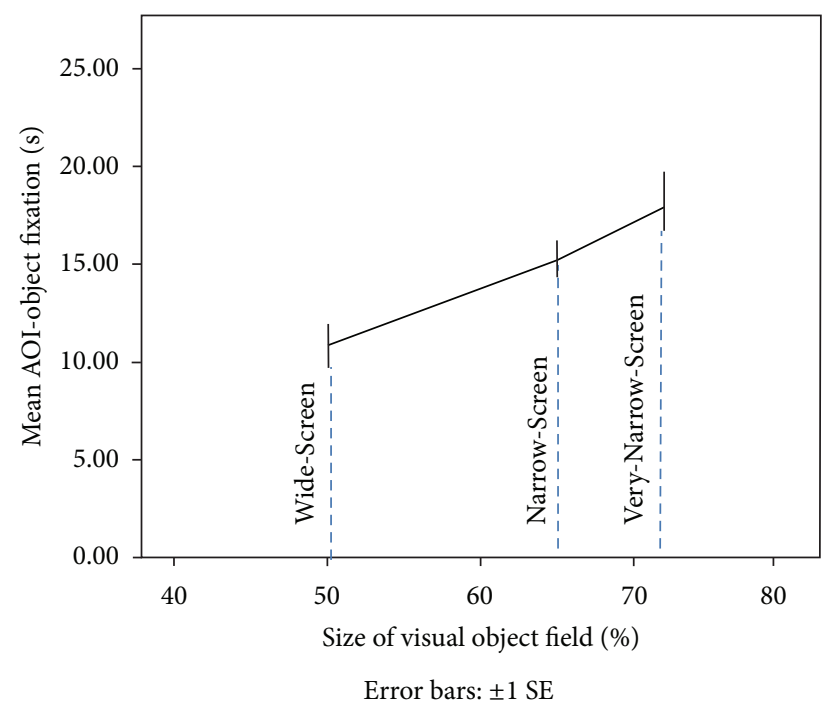

(b)

FIGURE 8: Graphic representation of the relation between size of the visual object field and Mean AOI-Total fixation time from eye tracking (a), and graphic representation of the relation between size of the visual object field and Mean AOI-objects fixation time from eye tracking (b).

we have obtained strongly converging results that partly replicate the results from the wide-screen/narrow-screen design originally devised by Wilcox and Baillargeon [4]. On the one hand, both methodologies showed significantly longer looks for the 9.5-month-old infants in the NarrowTest condition relative to the Wide-Test condition (as well as significantly longer looks for the 9.5-month-old infants in the Very-Narrow-Test condition compared to the Widetest condition). On the other hand-and contrary to the rationale behind the reference experiment-there was no evidence in the data that the infants used the available featural information to discriminate between the objects involved. We cannot conclude that the infants did not discriminate, but there is no indication in the data that the infants made use of any discriminatory information when parsing the events.

According to our original screen hypotheses regarding how infants should interpret the Narrow-Test event, the data seem to support the interpretation favored by $\mathrm{Xu}$ and her collaborators $[8,9]$. However, for two reasons, we do not believe that Xu's interpretation offers the best account of the data. First, Xu's interpretation is based on the assumption that the infants detect a change in appearance (i.e., from ball to box), and our data does not support this claim. Second, the data from our follow-up experiment, in which adults watched the looped movie clip of the Narrow-Test event, seem to question the whole reasoning behind the narrow-screen design, as well as the two interpretations currently offeredat least among adults.

Consequently, we believe the data warrants a third interpretation. We propose that, in the first year of their lives, infants' looking behavior across the four conditions in the present experiment might best be explained by stating that moving objects operate as strong attractors. This postulate leads to the prediction that, in a visual tracking task with a centrally located occluder, infants' looking times will, with all things being equal, be a linear function of the VOF. We further propose that, in such tasks, infants below 12 months of age will be fairly insensitive to "object changes" while being occluded. We dub this interpretation The Moving Object as Attractor (MOA) hypothesis.

In the following, we will discuss our findings and the MOA hypothesis in relation to previous studies using the wide-screen/narrow-screen design, as well as in relation to alternative interpretations of the original widescreen/narrow-screen design.

5.1. The MOA Hypothesis and Control Experiments Using Smaller Objects. In the reference paper by Wilcox and Baillargeon [4, Exp. 3], the authors discussed a simple alternative interpretation of the results from the wide-screen/narrowscreen design. The infants might simply be demonstrating a preference for looking at the narrow screen relative to the wide screen. In order to rule out this possibility, the authors conducted an elegant control experiment: the widescreen/narrow-screen design was replicated with a smaller set of objects, where the combined width of the objects did not surpass the width of the narrow screen. The reasoning was that, if the infants' longer looks in the reference experiment were caused by a "cognitive" surprise reaction due to a detection of a physical violation, this pattern of results should not manifest itself in the control, narrow-screen condition, employing smaller objects. That was exactly what the results showed [4, Exp. 3].

Eye tracking methodology seems ideally suited for testing the simple alternative interpretation that infants might simply prefer the narrow screen to the wide screen. As already reported, and as is evident from Figure 5, the screen width had no impact on the time devoted to looking at the screens 
(whereas screen width clearly influenced looking time on VOFs). Thus, the results from our study are in accordance with the control experiment in the reference study, with respect to ruling out the interpretation suggesting a simple preference for narrow screens.

The MOA hypothesis might seem to predict that the control experiment offered by Wilcox and Baillargeon [4] should have led to results similar to those obtained in the experimental condition in the reference study. This prediction is based on the assumption that smaller screens, all things equal, will imply larger VOFs. But because of the use of smaller objects in the control condition of the reference study, all things are not equal. Another possibility favored by the present authors is that smaller objects simply act as weaker attractors than do larger objects and consequently minimize the impact of using smaller screens. We did not replicate the control experiment with smaller objects, so we are unable to provide empirical evidence with respect to the original control experiment. However, recent evidence [21] provides indirect support for this alternative interpretation: using the classical design offered by $\mathrm{Xu}$ et al. [10], we wanted to test whether 12-month-old infants were able to individuate conceptually different but similarly-shaped objects borrowed from Pauen [22]. The objects taken from Pauen [22] were significantly smaller than the objects used in the original experiment by $\mathrm{Xu}$ et al. [10], and, in pilot studies, we were unable to replicate the original findings. Only after having reduced the screen sizes, in order to make the objects relatively more prominent in the display, were we [21, page 91] able to replicate the basic finding from Xu et al.s [10] study. Exactly the same effect might have been present in the control narrow screen condition in the reference study.

\subsection{The MOA Hypothesis and Previous Single Object Narrow-} Screen Control Experiments. The direct Narrow-Control test that we employed has been used in studies by Wilcox and her collaborators as well. For example, in their followup to the reference study, Wilcox and Baillargeon [5] obtained consistently shorter looking times in the narrow-screen ballball conditions (7.5-month-olds: $M_{\text {narrow-ball-ball }}=18.5 \mathrm{~s}$; $\mathrm{SD}=11.0 ; 4.5$-month-olds: $M_{\text {narrow-ball-ball }}=22.9 \mathrm{~s}, \mathrm{SD}=$ 9.2), relative to the narrow-screen ball-box conditions (7.5months-olds: $M_{\text {narrow-ball-box }}=34.2 \mathrm{~s}, \mathrm{SD}=8.6 ; 4.5$-montholds: $M_{\text {narrow-ball-box }}=40.2 \mathrm{~s}, \mathrm{SD}=10.5$; see also [23]). How should we understand these results in relation to the clearly diverging results obtained in the present study?

Let us consider the design differences. First, in the reference experiment, the objects were moved manually, whereas the objects in our experiment were moved mechanically by computer-controlled step motors. Second, all the singleobject control conditions used by Wilcox and colleagues have involved only one of the two objects, namely, the ball, whereas the infants in our Narrow-Control condition were tested in either a ball-ball or a box-box condition. Third, besides using the SLTP, we used eye tracking as an additional methodology. The most important of these differences may be that our design involved mechanized/artificial sounds, whereas the reference design did not. However, for two reasons, we do not believe that this difference could be responsible for the contrasting results.

First, in the large number of studies where different but related aspects of infants' object tracking abilities have been investigated, a variety of different "object transport systems" have been used. In some studies, objects have been moved by hand, either visibly ([4, Exp. 7 and 8$]$ and $[24,25]$ ) or invisibly ([4, Exp. 1-6] and [3, 5, 10, 26, 27]). In other studies, the movements have been conducted by using lever controlled carriers [28-31]. And in other studies still, objects have been moved by mechanical (motor driven) means [12, 13, 21, 32]. Finally, some have presented their movements using computer displays (e.g., [33, 34]). To the best of our knowledge, these different means of moving objects have not, by themselves, resulted in markedly different results. This is probably due to the fact that infants quickly learn and accommodate to how "self-propelled" objects move [35].

Second, other specific studies have replicated, through use of a mechanized version [21], a design that was originally carried out through manual means [10]. In this case, similar results were obtained across the two methodologies [21].

We admit that we cannot provide any firm explanation as to why we obtained longer looking times in our NarrowControl condition, relative to existing studies from Wilcox and Baillargeon's lab. Given the findings cited above, we do not, however, consider it likely that our mechanized and computer-controlled version should be less reliable than the standard procedures used in the reference design(s).

5.3. General Discussion of the MOA Hypothesis. Considering the fact that infants do indeed discriminate between, say, colors, as early as in the first months of life (e.g., [36, 37]), one might ask why older infants do not use this discriminatory ability when parsing the events in question?

Our answer to this question is twofold. First, this phenomenon is actually not rare at all in the infant research literature. For example, when two distinct and differentlooking stationary objects, after having been seen placed apart, subsequently are placed adjacently, three-month-old infants appear to perceive the visual test array as consisting of one single object [38], even though the infants are known to possess both shape constancy [39] and size constancy [40] at this age. As another example, infants of 10 months of age should easily be able to discriminate between exemplars from two clearly distinct object categories (e.g., [41]). However, Xu and Carey [3] showed that 10-month-old infants only use this information when spatiotemporal information about the number of objects present was unambiguous (the spatiotemporal condition), but not when spatiotemporal information was ambiguous (the property/kind condition). Thus, being unable, under some conditions, to demonstrate a given ability that works flawlessly in other conditions is not uncommon in infant research. Given the clear and systematic data obtained, this may also be the case in the present study.

Another core feature of the MOA hypothesis-the idea that moving objects are strong attractors for infants-is not revolutionary at all, but appears to be a central feature of infant perception $[42,43]$. 
The MOA hypothesis is also in accordance with the results obtained using the basic design employed by $\mathrm{Xu}$ and Carey [3] and the considerable number of replications that have followed using this design (e.g., $[10,21,26,27,44])$. The results from these experiments indicate that, in the first year of their lives, infants do not use featural information to individuate objects in this specific design. The basic characteristics of this design may be viewed as corresponding to a widescreen trial in the wide-screen/narrow-screen design, and, as predicted by the MOA hypothesis, infants below 12-months of age may be relatively insensitive to featural changes when tracking objects. Likewise, the MOA hypothesis is generally in accordance with the framework offered by Leslie et al. [45], where infants are believed to possess two basic systems, a "where" (a spatiotemporally-driven system) and a "what" system (a feature-driven system). The account offered by Leslie and colleagues is based on the assumption-shared by the MOA hypothesis - that, in the beginning of their lives, infants are far more sensitive to spatiotemporal information than featural information.

Part of the divergence between the results obtained in the present study and some of the prior results attained in widescreen/narrow-screen design studies remains unexplained for the time being. As argued here, we believe that the results from the present experiment cannot be satisfactorily explained with the existing theories but requires a new interpretation-what we have dubbed the MOA hypothesis. Besides offering a simple, yet encompassing, interpretation of the data presented here, the MOA hypothesis possesses two strengths that any good hypothesis should display. The MOA hypothesis is simple, and it is falsifiable.

The MOA hypothesis is parsimonious. The MOA hypothesis is based on, and in accordance with, well-documented infant cognitive research (see above), and no "rich" interpretations of infants' abilities - which at times may be difficult to test (e.g., [46]) - may be needed. While the principle of parsimony does not in any way guarantee a valid theory, the principle is healthy [47] and should at least be taken into consideration when choosing between different alternative theories.

A second crucial strength of the MOA hypothesis lies in the fact that it leads to very specific predictions about looking times in visual tracking tasks with a centrally located occluder. Whereas proposing and demonstrating a significant linear relationship between two data points is trivial, a demonstration of a linear relationship between three or more data points, as evidenced in the present experiment, is not. Hence, in order to test the MOA hypothesis, we propose a new series of replication experiments based on objects and procedures employed in the original wide-screen/narrowscreen design by Wilcox and Baillargeon [4]. The new experiments should use test screen sizes of the following widths: $33 \mathrm{~cm}, 30 \mathrm{~cm}, 17 \mathrm{~cm}$, and $14 \mathrm{~cm}$. All four test screens should be employed with "different-object" conditions (ball-box), and the two narrow-screens (width: $17 \mathrm{~cm}$ and $14 \mathrm{~cm}$ ) should also be used for additional "same-object" control conditions (ballball or box-box). Participants should be groups of infants from 4.5 months of age to 9.5 month of age. In order to take full advantage of the eye tracking methodology, we suggest that the events are presented on a monitor. According to Wilcox and Baillargeon's [4] account, the looking times of the infants should result in a significant qualitative shift (i.e., longer looks) when going from wide-screen conditions (regardless of wide-screen width) to narrow-screen "different objects" conditions (regardless of narrow-screen width). Furthermore, infants in all age groups should look reliably longer at the narrow-screen "different-objects" conditions, relative to the narrow-screen "same-object" control conditions.

In contrast, the MOA hypothesis would predict that the looking times obtained from infants above 6 months of age should display a significantly linear function of VOF, regardless of whether the conditions are with "different" or "same" objects.

Considering the potential consequences for our understanding of infant cognition in general, and object individuation in particular, we firmly believe that the data obtained in the present study warrants such further replication studies.

\section{Conclusion}

The results from the present experiment are important in at least three different ways: first, the results are important with regard to methodological development. By using two methodologies simultaneously, we have achieved the possibility of assessing their intermethodology reliability. Meanwhile, the use of eye tracking has allowed us to look into what Aslin [17, 18] has dubbed the microstructure of infants' visual attention, which became evident in the analysis where we split the infants' visual field into smaller subsections (i.e., screens and VOFs).

Second, the results are important because they lead to a very different, yet simple, interpretation of infants' looking behavior in the wide-screen/narrow screen design. By means of using three different screen widths in the test trials, we demonstrated the presence of a significant linear trend between the size of the VOF and the infants' looking time. Existing wide-screen/narrow-screen studies seem to have overlooked this possibility, probably, because none of the previous studies have collected more than two data points in the same study. By introducing the MOA hypothesis, we have offered a new and simple interpretation of the findings of the present study, as well as those of some (but not all) of the previous studies using the wide-screen/narrow-screen design. Our hypothesis yields clear, quantifiable predictions for future experiments.

Finally, the ambiguous and inconclusive results obtained in our followup experiment on an adult sample cast doubt the basic reasoning behind the original version of the widescreen/narrow-screen design. Employing a design where the key condition (the narrow-screen event) has no clear interpretation among adults should, at the very least, warrant caution.

Reaching consensus about the most convincing interpretation of the wide-screen/narrow-screen design will not only solve an important dispute within the field of object individuation. Because object individuation can be seen as a "general purpose tool" [2], having substantial impact on how infants 
conceive and think about objects [48] and subsequently how objects are given names [49], a firm solution to this dispute is therefore likely to contribute to our broader understanding of infants' cognitive development.

\section{Acknowledgments}

The research was supported by two Grants to the first author from the Danish Research Council for the Humanities (nos. 273-05-0182 and 273-07-0009), the Danish National Research Foundation (DNRF93), and MINDLab. The authors would like to thank Bo Sommerlund for software, statistical, and technical assistance and Lars Krogh and Niels Dynæs Hansen for constructing and maintaining our mechanized puppet theatres. The authors thank Kim Plunkett for stimulating discussions of the results and Trine Sonne and Jonathan Koppel for their comments on earlier versions of the paper. The authors would also like to thank Jytte Krøjgaard for the theatre curtains, Ditte Norup, Cecilie Kousholt, Johanne Uhd, and Ida Marie Lautrup for their indispensable help with phoning and data collection, and, finally, all participating infants and parents for making the study possible at all.

\section{References}

[1] P. Krøjgaard, "A review of object individuation in infancy," British Journal of Developmental Psychology, vol. 22, no. 2, pp. 159-183, 2004.

[2] P. Krøjgaard, "The human ability to single out and track specific objects trough space and time: origin and application," in Human Characteristics: Evolutionary Perspectives on Human Mind and Kind, H. Høgh-Olesen, P. Bertelsen, and J. Tønnesvang, Eds., pp. 89-116, Cambridge Scholars Publishing, 2009.

[3] F. Xu and S. Carey, "Infants' metaphysics: the case of numerical identity," Cognitive Psychology, vol. 30, no. 2, pp. 111-153, 1996.

[4] T. Wilcox and R. Baillargeon, "Object individuation in infancy: the use of featural information in reasoning about occlusion events," Cognitive Psychology, vol. 37, no. 2, pp. 97-155, 1998.

[5] T. Wilcox and R. Baillargeon, "Object individuation in young infants: further evidence with an event-monitoring paradigm," Developmental Science, vol. 1, no. 1, pp. 127-142, 1998.

[6] J. G. Bremner, "Cognitive development: knowledge of the physical world," in Wiley-Blackwell Handbook of Infant Development. Volume I: Basic Research, J. G. Bremner and T. D. Wachs, Eds., pp. 204-242, Wiley Blackwell, Chichester, UK, 2nd edition, 2010.

[7] A. Needham and R. Baillargeon, "Infants' use of featural and experiential information in segregating and individuating objects: a reply to Xu, Carey and Welch (2000)," Cognition, vol. 74, no. 3, pp. 255-284, 2000.

[8] F. Xu and S. Carey, "The emergence of kind concepts: a rejoinder to Needham and Baillargeon (2000)," Cognition, vol. 74, no. 3, pp. 285-301, 2000.

[9] F. Xu, "The development of object individuation in infancy," in Progress in Infancy Research, J. Fagan and H. Haynes, Eds., vol. 3, pp. 159-192, Lawrence Erlbaum, Mahwah, NJ, USA, 2003.

[10] F. Xu, S. Carey, and N. Quint, "The emergence of kind-based object individuation in infancy," Cognitive Psychology, vol. 49, no. 2, pp. 155-190, 2004.
[11] R. Baillargeon, "Innate ideas revisited: for a principle of persistence in infants'physical reasoning," Perspectives on Psychological Science, vol. 3, pp. 2-13, 2008.

[12] P. Krøjgaard, "Comparing infants' use of featural and spatiotemporal information in an object individuation task using a new event-monitoring design," Developmental Science, vol. 10, no. 6, pp. 892-909, 2007.

[13] P. Krøjgaard, "A direct comparison of infants' use of featural and spatiotemporal information when individuating objects in a non-occlusion event-monitoring design," European Journal of Developmental Psychology, vol. 6, no. 6, pp. 641-665, 2009.

[14] R. Brown, "How shall a thing be called?" Psychological Review, vol. 65, no. 1, pp. 14-21, 1958.

[15] M. J. Peltola, J. M. Leppänen, V. K. Vogel-Farley, J. K. Hietanen, and C. A. Nelson, "Fearful faces but not fearful eyes alone delay attention disengagement in 7-month-old infants," Emotion, vol. 9, no. 4, pp. 560-565, 2009.

[16] P. C. Quinn, M. M. Doran, J. E. Reiss, and J. E. Hoffman, “Time course of visual attention in infant categorization of cats versus dogs: evidence for a head bias as revealed through eye tracking," Child Development, vol. 80, no. 1, pp. 151-161, 2009.

[17] R. N. Aslin, "What's in a look?" Developmental Science, vol. 10, no. 1, pp. 48-53, 2007.

[18] R. N. Aslin, "Infant eyes: a window on cognitive development," Infancy, vol. 17, no. 1, pp. 126-140, 2012.

[19] T. Wilcox and C. Chapa, "Priming infants to attend to color and pattern information in an individuation task," Cognition, vol. 90, no. 3, pp. 265-302, 2004.

[20] S. Carey and F. Xu, "Infants' knowledge of objects: beyond object files and object tracking," Cognition, vol. 80, no. 1-2, pp. 179-213, 2001.

[21] O. S. Kingo and P. Krøjgaard, "Object manipulation facilitates kind-based object individuation of shape-similar objects," Cognitive Development, vol. 26, no. 2, pp. 87-103, 2011.

[22] S. Pauen, "Evidence for knowledge-based category discrimination in infancy," Child Development, vol. 73, no. 4, pp. 1016-1033, 2002.

[23] T. Wilcox, "Object individuation: infants' use of shape, size, pattern, and color," Cognition, vol. 72, no. 2, pp. 125-166, 1999.

[24] T. Wilcox and A. Schweinle, "Object individuation and event mapping: developmental changes in infants' use of featural information," Developmental Science, vol. 5, no. 1, pp. 132-150, 2002.

[25] R. J. Woods, T. Wilcox, J. Armstrong, and G. Alexander, "Infants' representations of three-dimensional occluded objects," Infant Behavior and Development, vol. 33, no. 4, pp. 663-671, 2010.

[26] P. Krøjgaard, "Object individuation in 10-month-old infants: do significant objects make a difference?” Cognitive Development, vol. 15, no. 2, pp. 169-184, 2000.

[27] P. Krøjgaard, "Object individuation in 10-month-old infants: manipulating the amount of introduction," British Journal of Developmental Psychology, vol. 21, no. 3, pp. 447-463, 2003.

[28] A. Aguiar and R. Baillargeon, "2.5-month-old infants' reasoning about when objects should and should not be occluded," Cognitive Psychology, vol. 39, no. 2, pp. 116-157, 1999.

[29] A. Aguiar and R. Baillargeon, "Developments in young infants' reasoning about occluded objects," Cognitive Psychology, vol. 45, no. 2, pp. 267-336, 2002.

[30] Y. Luo and R. Baillargeon, "Can a self-propelled box have a goal?-psychological reasoning in 5-month-old infants," Psychological Science, vol. 16, no. 8, pp. 601-608, 2005. 
[31] Y. Luo and R. Baillargeon, "When the ordinary seems unexpected: evidence for incremental physical knowledge in young infants," Cognition, vol. 95, no. 3, pp. 297-328, 2005.

[32] Y. Luo, L. Kaufman, and R. Baillargeon, "Young infants' reasoning about physical events involving inert and self-propelled objects," Cognitive Psychology, vol. 58, no. 4, pp. 441-486, 2009.

[33] J. G. Bremner, S. P. Johnson, A. Slater et al., "Conditions for young infants' perception of object trajectories," Child Development, vol. 76, no. 5, pp. 1029-1043, 2005.

[34] D. Mareschal and M. H. Johnson, "The "what" and "where" of object representations in infancy," Cognition, vol. 88, no. 3, pp. 259-276, 2003.

[35] K. Shutts, L. Markson, and E. S. Spelke, “The developmental origins of animal and artifact concepts," in The Origins of Object Knowledge, B. M. Hood and L. R. Santos, Eds., pp. 189-210, Oxford University Press, Oxford, UK, 2009.

[36] P. J. Kellman and M. Banks, "Infant visual perception," in Handbook of Child Psychology: Vol. 2. Cognition, Perception and Language, W. Damon, D. Kuhn, and R. Siegler, Eds., pp. 103-146, Wiley, New York, NY, USA, 5th edition, 1998.

[37] D. Y. Teller, "Spatial and temporal aspects of infant color vision," Vision Research, vol. 38, no. 21, pp. 3275-3282, 1998.

[38] E. S. Spelke, "Perception of unity, persistence, and identity," in Neonate Cognition: Beyond the Blooming Buzzing Confusion, J. Mehler and R. Fox, Eds., pp. 89-113, Erlbaum, Hillsdale, NJ, USA, 1985.

[39] A. Slater and V. Morison, "Shape constancy and slant perception at birth," Perception, vol. 14, no. 3, pp. 337-344, 1985.

[40] A. Slater, A. Mattock, and E. Brown, "Size constancy at birth: newborn infants' responses to retinal and real size," Journal of Experimental Child Psychology, vol. 49, no. 2, pp. 314-322, 1990.

[41] P. C. Quinn and P. D. Eimas, "Perceptual organization and categorization in young infants," Advances in Infancy Research, vol. 10, pp. 1-36, 1996.

[42] M. E. Arterberry, L. G. Craton, and A. Yonas, "Infants' sensitivity to motion-carried information for depth and object properties," in Visual Perception and Cognition in Infancy, C. E. Granrud, Ed., pp. 215-234, Erlbaum, Hillsdale, NJ, USA, 1993.

[43] P. W. Jusczyk, S. P. Johnson, E. S. Spelke, and L. J. Kennedy, "Synchronous change and perception of object unity: evidence from adults and infants," Cognition, vol. 71, no. 3, pp. 257-288, 1999.

[44] L. Bonatti, E. Frot, R. Zangl, and J. Mehler, "The human first hypothesis: identification of conspecifics and individuation of objects in the young infant," Cognitive Psychology, vol. 44, no. 4, pp. 388-426, 2002.

[45] A. M. Leslie, F. Xu, P. D. Tremoulet, and B. J. Scholl, "Indexing and the object concept: developing 'what' and 'where' systems," Trends in Cognitive Sciences, vol. 2, no. 1, pp. 10-18, 1998.

[46] M. M. Haith and J. B. Benson, "Infant cognition," in Handbook of Child Psychology, W. Damon, D. Kuhn, and R. Siegler, Eds., vol. 2, pp. 199-254, Wiley, New York, NY, USA, 1998.

[47] M. M. Haith, "Who put the cog in infant cognition? Is rich interpretation too costly?" Infant Behavior and Development, vol. 21, no. 2, pp. 167-179, 1998.

[48] T. Wilcox and T. R. Smith, "The development of infants' use of property-poor sounds to individuate objects," Infant Behavior and Development, vol. 33, no. 4, pp. 596-604, 2010.

[49] E. S. Spelke, "Discussion, section C: the ontogeny of perceptual and causal knowledge," in Thought without Language, L. Weiskrantz, Ed., pp. 229-236, Clarendon Press/Oxford University Press, Oxford, UK, 1988. 

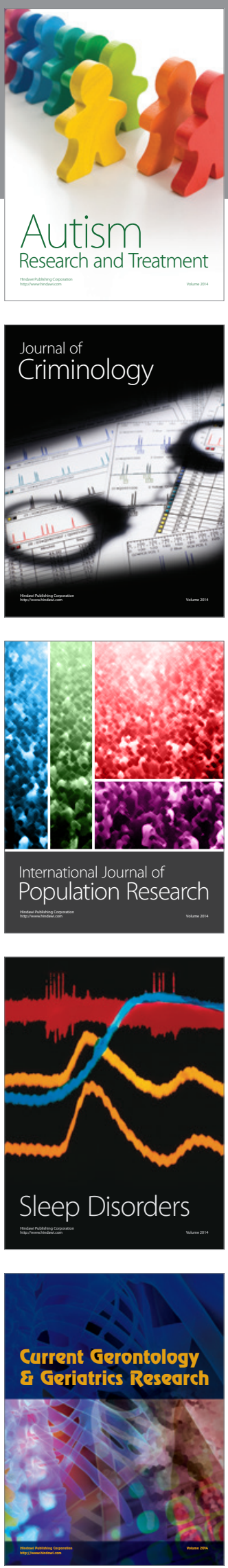
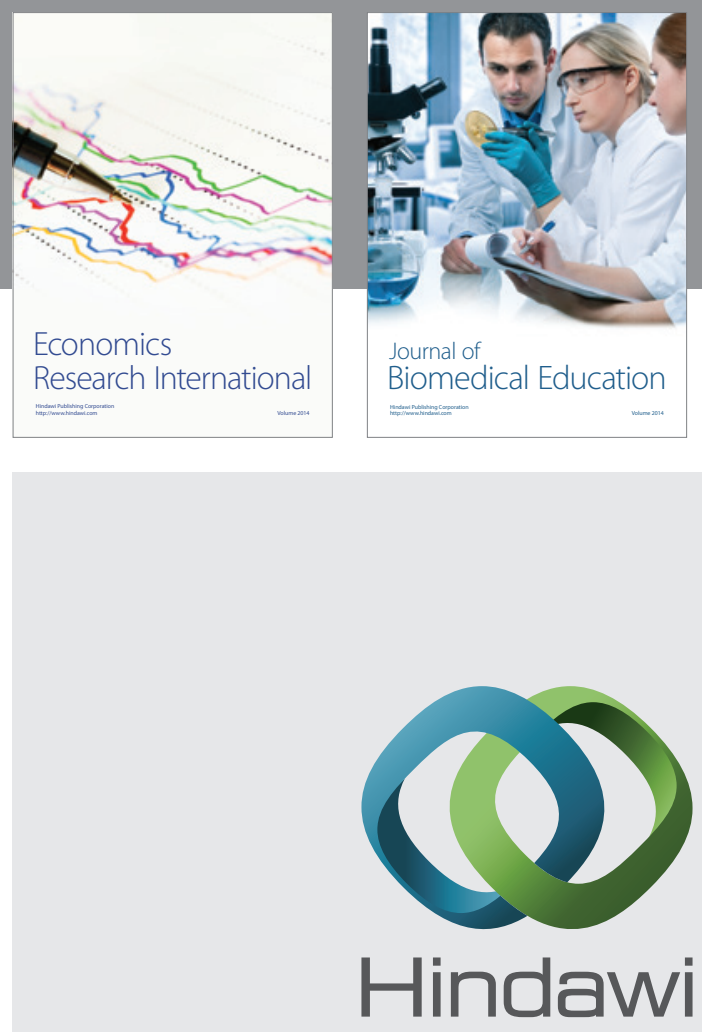

Submit your manuscripts at

http://www.hindawi.com
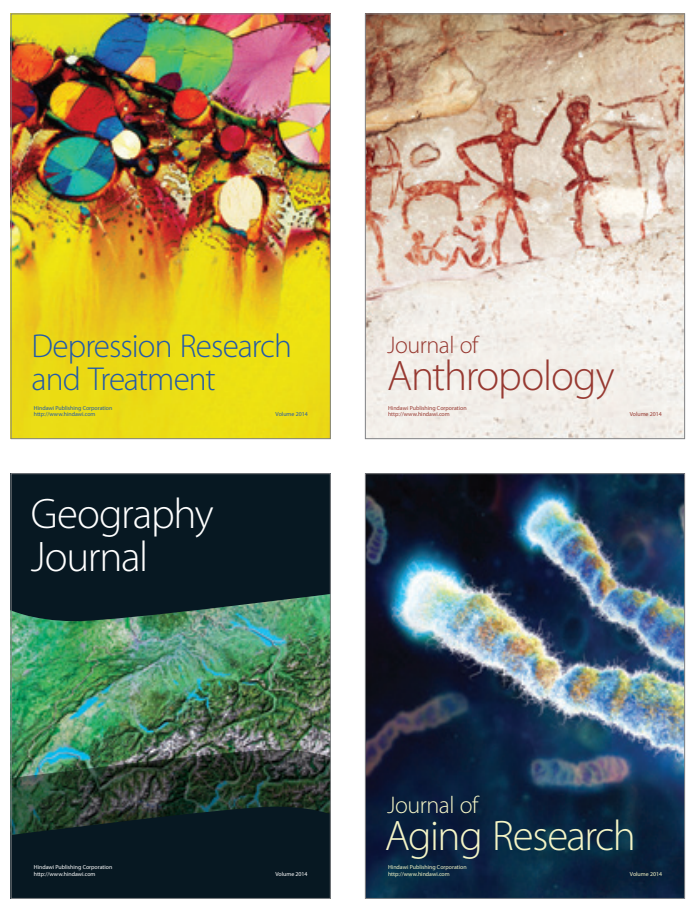
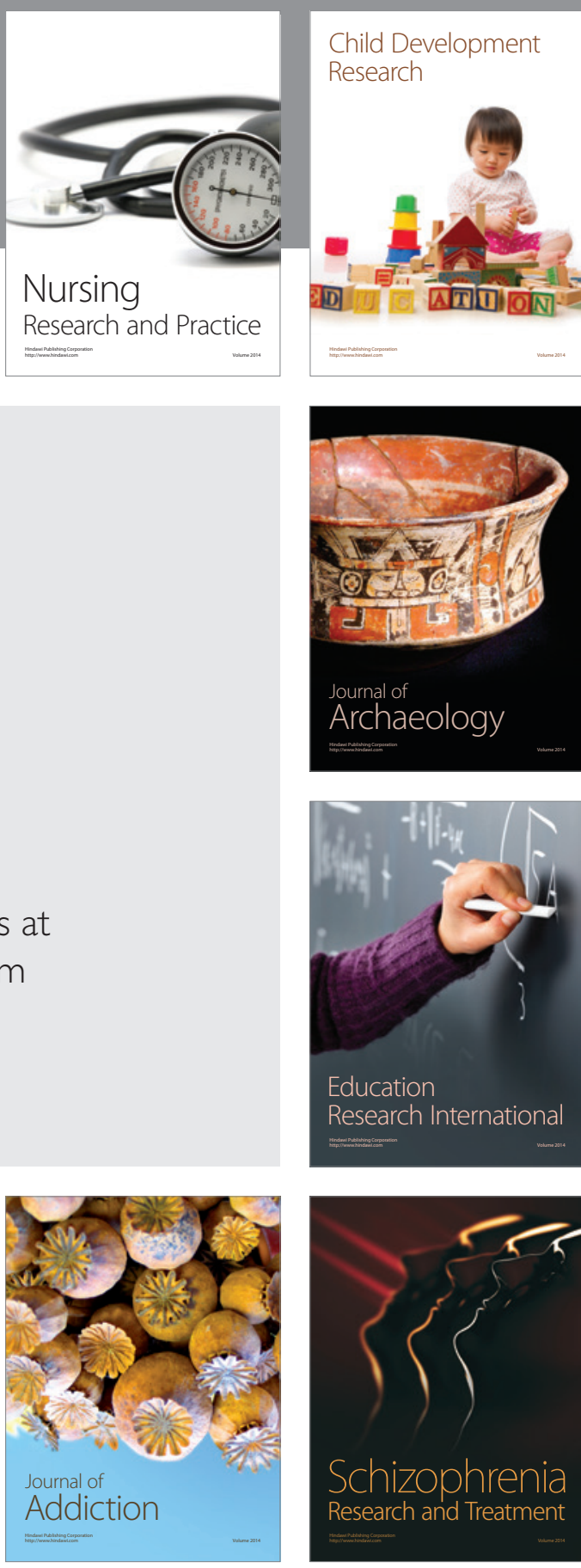

(D)
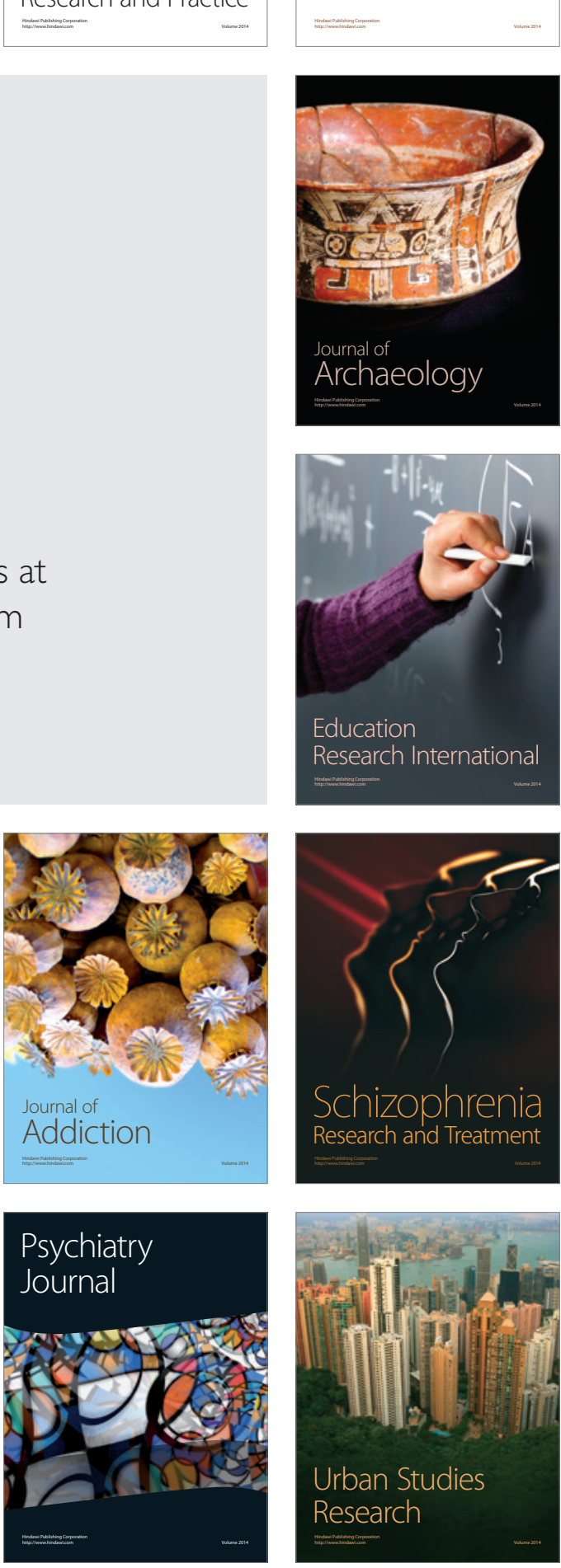